\title{
Alkylating Agent-Related Myelodysplastic Syndrome
}

National Cancer Institute

\section{Source}

National Cancer Institute. Alkylating Agent-Related Myelodysplastic Syndrome. NCI

Thesaurus. Code C7643.

A disorder seen following cancer chemotherapy. It is the most common cause of therapy-related myelodysplastic syndromes. It typically manifests several years after initiation of single or multi-agent chemotherapy with alkylators. Mutagenic potential of alkylating agents is believed to be age and cumulative dose-dependent. Deletions in chromosomes 5 and 7 are associated with susceptibility to this disorder. Clinical signs may include fatigue, dyspnea, bruising and frequent infections. Clinical course may progress to bone marrow failure or acute myeloid leukemia that is refractory to treatment. Prognosis is dismal with survivability usually less than one year. 